\title{
Slow and Fast Atomic Motion Observed by Aberration-Corrected STEM
}

\author{
O.L. Krivanek ${ }^{1}$, N. Dellby ${ }^{1}$, M.F. Murfitt ${ }^{1}$, Z.S. Szilagyi ${ }^{1}$, M.F. Chisholm ${ }^{2}$ and K. Suenaga ${ }^{3}$ \\ ${ }^{1}$ Nion Co., $11028^{\text {th }}$ St., Kirkland, WA 98033 \\ ${ }^{2}$ Oak Ridge National Laboratory, Mats Sci. and Techn. Division, Oak Ridge, TN 37831 \\ ${ }^{3}$ National Institute of Advanced Industrial Science and Technology, Tsukuba 305-8565, Japan
}

Annular dark field (ADF) imaging in an aberration-corrected STEM is now able to resolve single light atoms such as boron, carbon, nitrogen and oxygen with signal-to-noise ratios (SNRs) that are high enough to allow chemical identification of the atoms [1,2]. The high SNRs also make it possible to determine whether an atom was present or not at each individual image pixel, especially for heavy atoms. The required performance (probe size $\sim 1 \AA$; probe current $\sim 50 \mathrm{pA}$; detector DQE 1; stable total system that does not produce spurious "shakes") can now be achieved at primary voltages as low as $60 \mathrm{kV}$, which minimizes the beam-induced motion of the atoms. This has opened up a new window for studying the motion of single atoms, both slow and fast.

Fig. 1. shows an unprocessed sequence of images of a single wall carbon nanotube filled with Ercontaining nanopods, plus one STEM BF image, acquired with the Nion UltraSTEM ${ }^{\text {TM }}$ [3] at ORNL. The operating voltage was $60 \mathrm{kV}$, and the total dose about $2 \times 10^{6} \mathrm{e}^{-} / \AA^{2}$. Remarkably, the nanotube did not experience any visible radiation damage despite the large dose (equal to $3 \times 10^{3}$ $\mathrm{C} / \mathrm{cm}^{2}$ ). The nanopods inside the nanotube, however, did change under the beam, typically by merging together. The erbium atoms explored many positions within the nanopods, and some even escaped outside of the nanopods (e.g. the Er atom between nanopods 1 and 2).

The dose per image was progressively increased during the observation, from $4 \times 10^{4} \mathrm{e}^{-} / \AA^{2}$ for frames (a) and (b) to $2 \times 10^{5} \mathrm{e}^{-} / \AA^{2}$ for frames (f) to (h), by decreasing the pixel size but keeping the time per pixel constant at $10 \mu \mathrm{sec}$. In the higher exposure images, the atoms often moved while the beam was scanning over them. Three different types of motion are documented in Fig. 2 (and explained in greater detail in reference [1]): a rapid oscillation of the same Er atom between two positions at a frequency $>>100 \mathrm{kHz}$, which gave two atomic images, each one half as intense as the image of a stationary atom (Fig. 2a), an Er atom jumping between several sites in a small area, with a jump frequency of 100-1000 Hz, which gave alternating bright and dark image lines aligned with the line scan direction (Fig. 2d), and an Er atom that jumped twice while the beam was nearby but not on it, with the second jump taking it right under the beam. This resulted in a dark scan line running across the atom's image, the next bright scan line being displaced $3.5 \AA$ to the left, and then a return of the atom to its original location when the beam was at the arrowed pixel (Fig. 2e).

Observations such as the above require excellent long-term and short-term stabilities of the microscope. Fig. 3 explores the needed short-term stability by adding, in the computer, random probe "wiggles" of frequencies $0-800 \mathrm{~Hz}$ to the image of two relatively stable Er atoms of Fig. 1g. The atoms become unrecognizable at $1.0 \AA$ r.m.s. added probe wiggles, and are objectionably "squiggly" with wiggles of $0.25 \AA$. $0.1 \AA$ wiggles change the outer boundary of the nanotube in a detectable way. The experimental image is not disturbed only by added wiggles of $0.05 \AA$. This shows that we have now achieved an overall stability better than $0.1 \AA$ r.m.s., through measures such as an ultra-stable sample stage and ultra-stable power supplies for the optical elements. 
Research at ORNL was sponsored by DOE Office of Basic Sciences, Division of Mats Sci. and Eng. [1] O.L. Krivanek et al., Ultramicroscopy, accepted for publication (2010).

[2] O.L. Krivanek et al., Nature, accepted for publication (2010).

[3] O.L. Krivanek et al., Ultramicroscopy 108 (2008) 179.
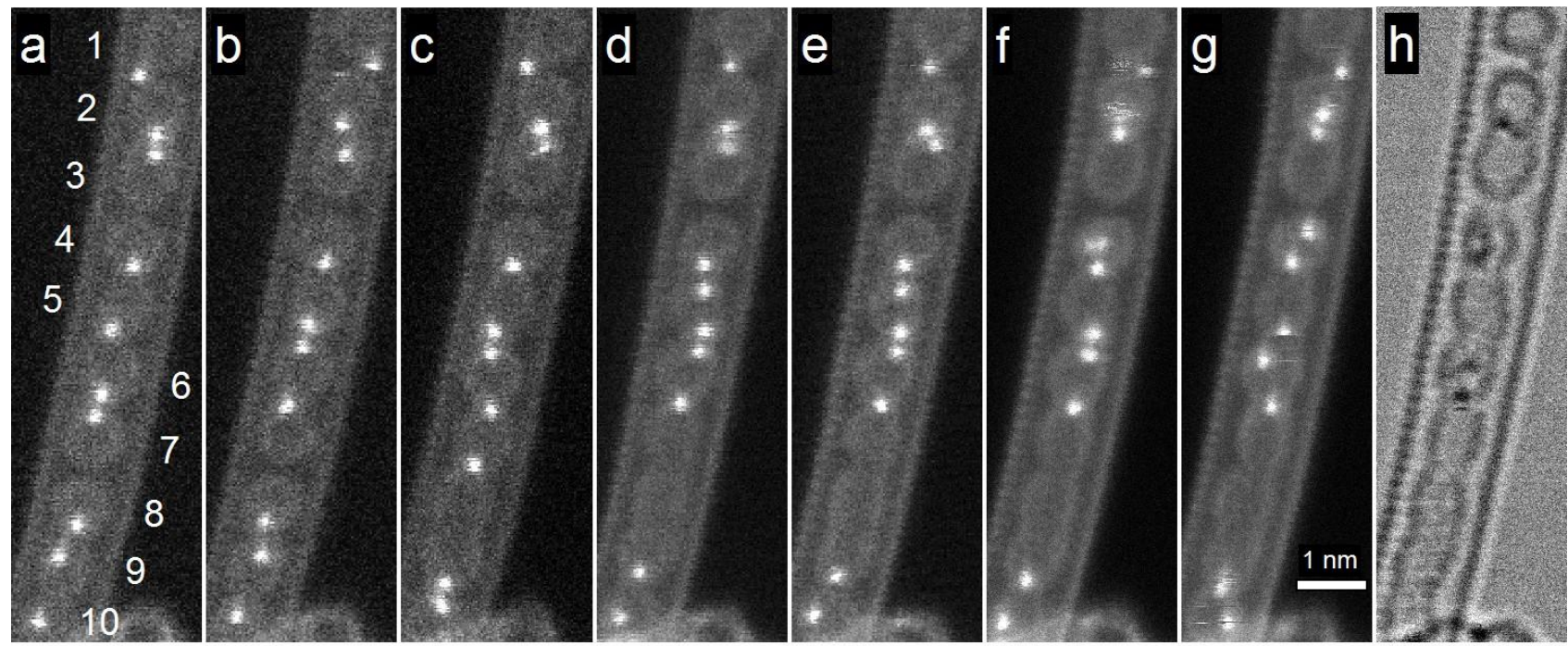

FIG. 1. Single wall C nanotube filled with Er-containing nanopods (numbered in a). a)-c) HAADF images (inner half-angle $\sim 80 \mathrm{mr}$, outer half-angle $\sim 200 \mathrm{mr}$ ); d)-g) MAADF images (inner half-angle $\sim 60 \mathrm{mr}$ ); h) BF image (collection half-angle $\sim 5 \mathrm{mr}$ ). All images acquired at $10 \mu \mathrm{sec}$ per pixel.
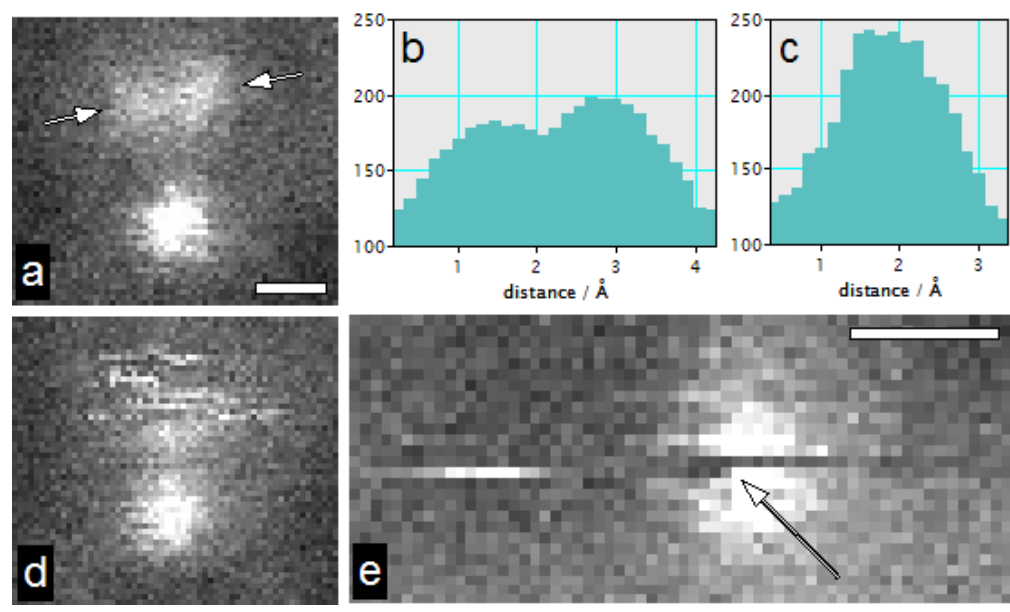

FIG 2. a) Er atoms in nanopod 4 in frame $1(\mathrm{f}) ; \mathrm{b})$ line profile taken between the arrows in (a); c) line profile of the bottom atom in (a), d) atoms at the boundary of nanopods 2 and 3 in frame (f); e) Er atom in nanopod 10 in frame (f). Scale bars in (a) and (e) are $2 \AA$; (d) has the same magnification as (a). Note that the observed "jumps" were only seen for the Er atoms - the nanotube walls showed no similar disturbances.

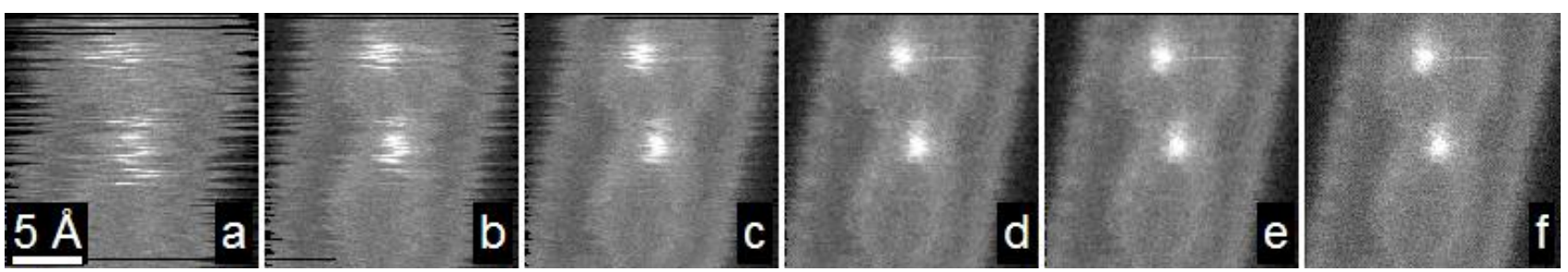

FIG 3. Images of nanopods 6+7 of Fig. $1 \mathrm{~g}$, with various amounts of added probe "wiggles". a) 1.0 $\AA$ r.m.s., b) $0.5 \AA$, c) $0.25 \AA$, d) $0.1 \AA$, e) $0.05 \AA$, f) experimental image. The horizontal streak to the right of the top atom in images d-f, which is due to the atom's motion, becomes undetectable in a-c. 